\title{
Prestack least-squares RTM on surface offset gathers for more reliable quantitative interpretation - Santos Basin case study
}

Maria Shadrina*, Maud Cavalca, Marcela Ortin, Marina Pantoja, Elena Medina, Claudio Leone and Robin Fletcher, Schlumberger

Copyright 2021, SBGf - Sociedade Brasileira de Geofísica

This paper was prepared for presentation during the $17^{\text {th }}$ International Congress of the Brazilian Geophysical Society held in Rio de Janeiro, Brazil, 16-19 August 2021.

Contents of this paper were reviewed by the Technical Committee of the $17^{\text {th }}$ International Congress of the Brazilian Geophysical Society and do not necessarily represent any position of the SBGf, its officers or members. Electronic reproduction or storage of any part of this paper for commercial purposes without the written consent of the Brazilian Geophysical Society is prohibited.

\section{Abstract}

The geological complexity of the Santos Basin, highly variant salt layers above the reservoir and limitations of the acquisition system lead to irregular illumination of the pre-salt target. This imposes a strong non-geological imprint on the seismic amplitudes; therefore, undermining and introducing uncertainty in further quantitative analysis. To handle the illumination challenges associated with a narrow-azimuth seismic data, prestack leastsquares reverse time migration (LSRTM) is applied. Herein, we discuss the workflow as well as the challenges associated with its practical application. We demonstrate that prestack LSRTM offers superior imaging, providing higher-resolution offset gathers with enhanced amplitude fidelity, leading to more reliable reservoir characterization.

\section{Introduction}

In the last decade, least-squares migration has emerged as a viable solution to address uneven subsurface illumination and improve imaging in the presence of complex overburden and/or limited acquisition geometries. Its image-domain implementation (Fletcher et al., 2012) has been applied to a large variety of geological settings and basins worldwide and demonstrated its potential to mitigate the non-geological imprint of the seismic experiment (Cavalca et al., 2019; Pereira-Dias et al., 2019). By bringing amplitudes closer to the true reflectivity, this approach enables more confident quantitative interpretation and analysis based on seismic data (Dias et al., 2019).

The Santos Basin, offshore Brazil, is home to large oil fields of very high commercial value. The complex geology of this province presents many challenges for imaging and characterization of the subsurface. The presalt reservoirs, which are the focus of the current study, are buried underneath highly variant salt layers that strongly distort the wave propagation and, coupled with the seismic acquisition geometry constraints, lead to variable illumination of the targets. The occurrence of igneous bodies intercalated in the post-salt, salt and presalt layers constitute additional challenges for the reservoir production (Penna et al., 2019).

To improve the image quality and the seismic data reliability for strategic decisions in developing the pre-salt reservoirs, the latest seismic processing technologies have been used. Imaging was performed using reverse time migration (RTM), which is the method of choice in complex salt environments. Provided that a sufficiently accurate earth model can be obtained, RTM enables accurate kinematic imaging of pre-salt events. Nevertheless, it is unable to fully restore the subsurface reflectivity amplitudes due to inadequate illumination. The data set under study is narrow azimuth (NAZ) towed streamer seismic data acquired in 2012 in the Santos Basin. To address the challenges of the geological complexity in the province and provide a trustworthy image of the NAZ data set, advanced velocity model building tools were adopted as part of the reprocessing sequence started in 2018 (Kang et al., 2019). The workflow was followed by a full-field $45 \mathrm{~Hz}$ prestack leastsquares reverse time migration (LSRTM) applied to surface offset gathers (SOGs).

Applying LSRTM to SOGs should mitigate the nongeological amplitude variations present in them, potentially enabling more accurate prestack quantitative analysis and enhanced estimation of elastic property distributions.

\section{Method}

In the framework of prestack LSRTM applied to RTM SOGs in the image domain, each SOG is regarded as the result of a non-stationary convolution of the subsurface reflectivity with a blurring operator. This operator is approximated through a grid of point spread functions (PSFs). Point spread functions can be regarded as an impulse response of the modelling and imaging experiment. PSFs capture space-, depth- and dip-variant illumination effects due to shortcomings of the acquisition system, complex overburden geology and limited bandwidth on the diffractor point in the subsurface (Fletcher et al., 2012). PSFs are explicitly modelled for each SOG, based on the velocity field and the source and receiver locations.

PSF generation is the most computationally intensive step in the LSRTM workflow and has foremost influence on the inversion accomplishing its potential. Therefore, much consideration should be given to the parameterization and quality control during PSFs modelling. In the context of RTM, accurate and high-resolution earth models enclosing high-velocity contrasts, such as salt bodies, are often required to derive the best kinematic image. Detailed velocity fields benefit the LSRTM workflow by more realistically representing the subsurface, hence, more accurately capturing the wave propagation of the seismic experiment and its illumination effects. However, working with rapidly varying velocity models presents additional challenges. PSFs are modelled under a single scattering assumption, whereas, the presence of strong velocity contrasts causes high-order scattering at the hard 
boundaries and generation of artefacts contaminating the neighboring PSFs (Cavalca et al., 2018). The contamination of PSFs by coherent multi-scattered energy (or other incoherent noise) in individual offset gathers becomes even more pronounced and problematic than for full image PSFs, which benefit from noise mitigation due to stacking. PSFs contaminated by strong multi-scattering artifacts can lead to poor inversion results and artefacts leaking into the reflectivity solution. Smoothing the velocity field would compromise the accuracy of the PSF modelling and cannot be envisaged; therefore, mitigation measures must be used to reduce their negative impact. In the current study, a combination of different approaches was adopted to address PSFs energy contamination effectively, including methods such as PSF encoding during modelling and PSF reconstruction at the salt boundaries prior to inversion (Cavalca et al., 2018).

Deconvolution of the blurring operator from the SOGs is achieved through least-squares optimization by seeking for the prestack (pseudo) reflectivity model that best minimizes the misfit between the migrated SOGs and their synthetic counterparts. For each point in the image, the blurring operator is approximated by interpolating the modelled PSFs. The inversion is performed for all SOGs simultaneously. Prior and sparsity constraints can be used in the inversion as well as additional regularization across offsets to ensure consistent reflectivity across neighboring gathers. Mild regularization across offsets was employed in the current study and proved to be very auspicious at addressing sparse noise in the SOGs and preventing its leakage into the reflectivity. Vigilant parameterization and quality control were performed to ensure preservation of the AVO response.

LSRTM was applied to the full-depth interval of $12 \mathrm{~km}$. The global inversion of this large area, coupled with the presence of energetic top salt events, required special attention during parameterization. Highly energetic events tend to dominate the inversion problem and can often jeopardize convergence in the (less energetic) target intervals. This effect was mitigated by using spatially variant weights that conform with the structural geology of the area and facilitate convergence in the reservoir.

\section{Results}

The geological complexity of the Santos Basin and limitations of the narrow azimuth acquisition lead to nonuniform illumination of the pre-salt reservoir. Figure 1 shows a seismic RMS maps extracted from RTM (1a) and LSRTM reflectivity (1b) images (SOGs full stacks) in the 1 $\mathrm{km}$ interval below the base of salt. The values distribution on the attribute maps derived from RTM and LSRTM images suggests a significant illumination footprint present in the RTM seismic amplitudes, with more balanced magnitudes throughout after LSRTM application.

Figure 2 displays the intersection line from the RTM image (2a) and LSRTM image (2b). Examination of the intersection lines strengthens this observation, showing the non-geological amplitude variations and dimming zones in the pre-salt interval located underneath thicker sections of the salt body (highlighted by the blue arrows) in the RTM image. The LSRTM image demonstrates effective mitigation of the illumination imprint. More equalized and consistent amplitudes can be observed along the main events as well as sharper imaging of the fault structures. The improved focusing of the events observed in the stacked image after prestack LSRTM can be partially attributed to deblurring of the offset gathers prior to stacking; therefore, potentially enhancing the image clarity.

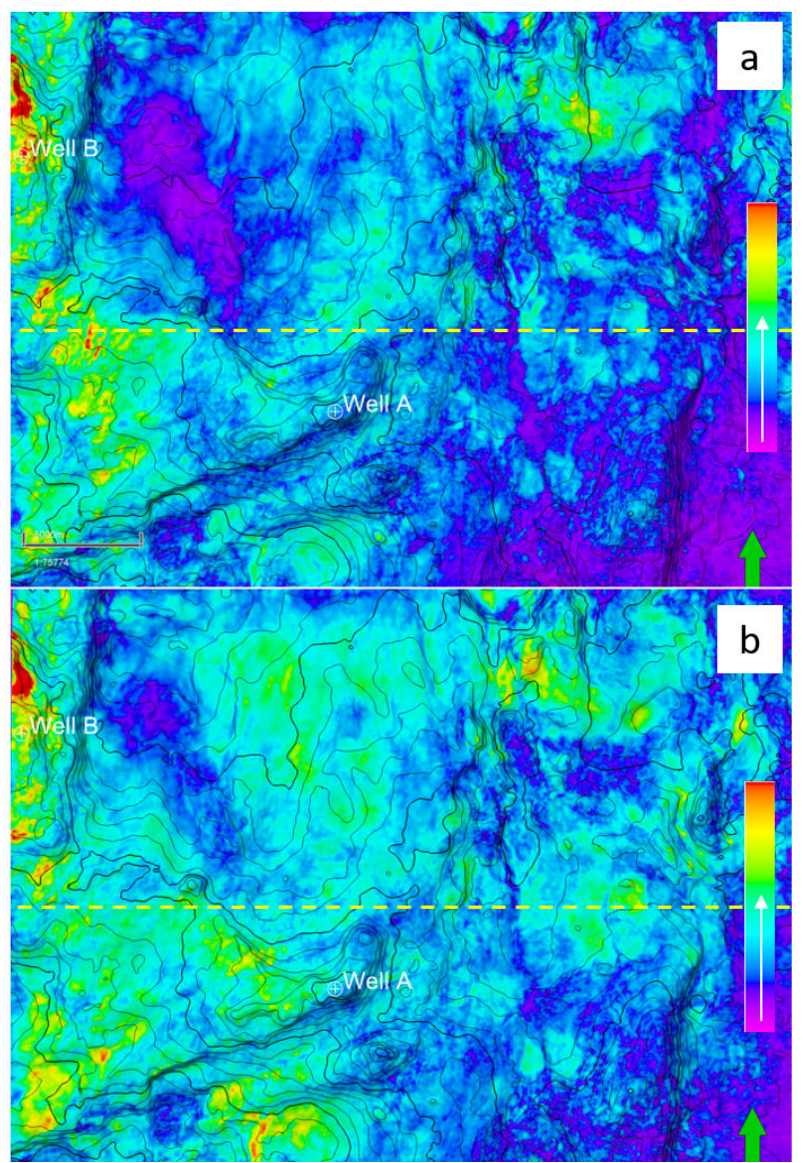

Figure 1 - RMS attribute maps extracted from the full RTM (a) and LSRTM (b) images in a $1 \mathrm{~km}$ depth window below the base of salt.

Besides the apparent improvement of the imaging and amplitude balancing in the full stack, reduction of the illumination footprint across offsets is also observed in the SOGs at Well A (Figure 3). RTM and LSRTM gathers are displayed alongside the synthetic seismic response modelled using the well reflectivity and 1D convolution with a representative wavelet. A few qualitative remarks can be made based on the comparison. Firstly, the LSRTM gathers show an improved event continuity with offset as well as an enhanced resolution. Moreover, the amplitudes of the events are in better agreement with the synthetic response, not only with offset, but also along the depth of the well. Figure 3 shows comparison between the events' AVO response with the modelled synthetic on an AVO cross plot for one of the events in the target interval, highlighted by the blue depth marker. The AVO response observed from LSRTM gathers matches the synthetic closer, validating the improvement in the fidelity of the amplitudes and reduction of the illumination distortion across offsets. 


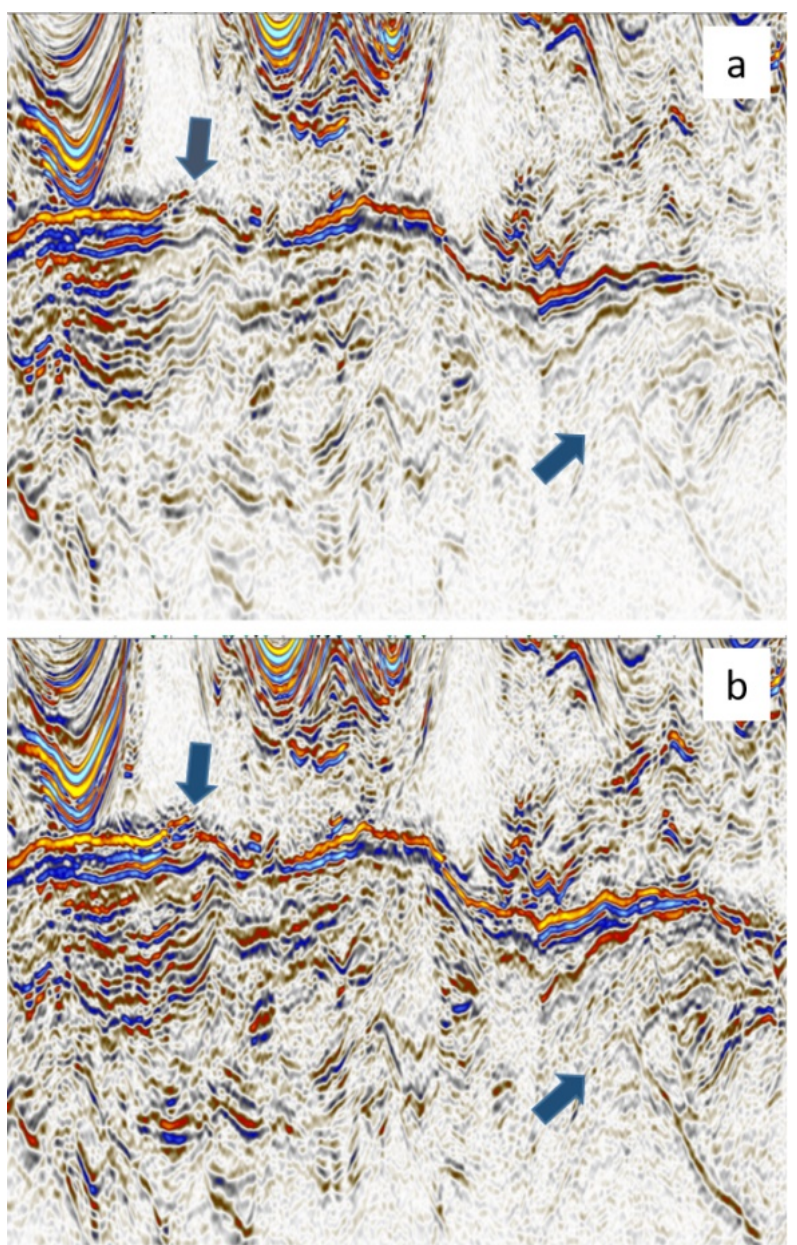

Figure 2 - Intersection line (location shown in Figure 1) from the RTM (a) and LSRTM (b) full stacks (SOGs stacked together).

Illumination variations in the subsurface and across offsets can have a substantial impact on estimating the elastic properties and on seismic reservoir characterization. To further evaluate and visualize the effect of the illumination compensation, quick relative AVO inversions were performed using both RTM and LSRTM angle gathers.

The main objective of the AVO inversions was quality control of the process and comparison between the two sets of gathers; hence, all inversion parameters were consistent between the tests, with angle-dependent wavelets extracted at Well A from the same window. Figures 4 and 5 display the seismic image and the inversion solutions derived for the intersection line extending from Well A to B. Amplitudes balancing is evident in the seismic sections (Figures $4 a$ and $5 a$ ) and is directly translated into the relative acoustic impedance (Figures $4 \mathrm{~b}$ and $5 \mathrm{~b}$ ), showing a different, more coherent property distribution as well as a broader bandwidth solution. As expected, the inversion solutions are very similar at Well A, which was used for wavelet extraction. They are also consistent at Well B because the wells are in areas that do not suffer from significant illumination issues, as can be seen in Figures 1a and $1 \mathrm{~b}$.

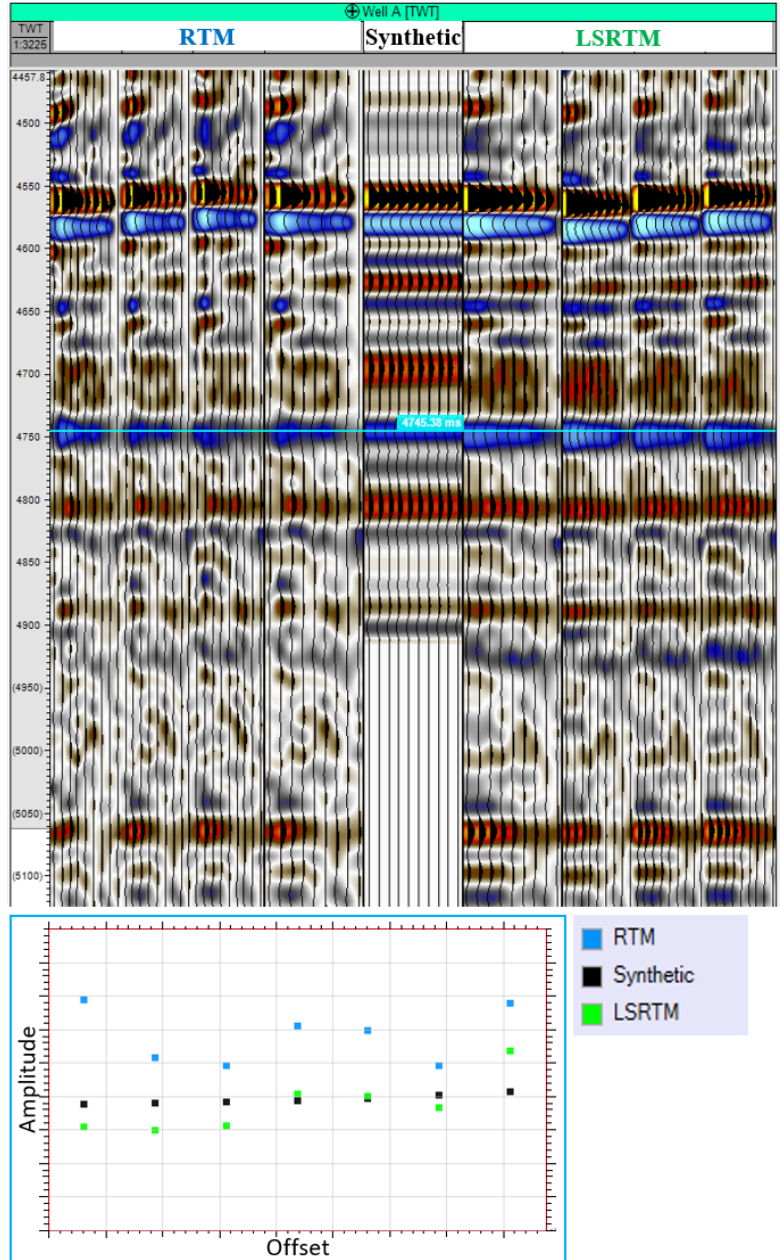

Figure 3 - RTM (left) and LSRTM (right) SOGs at Well A, with modeled synthetic response based on the well reflectivity (center) and an AVO analysis cross plot (bottom) for the event highlighted by the blue depth marker.

Two shadow zones present in the seismic data, due to a thicker salt layer in the overburden, appear to correspond to the decrease in the dynamic range of the Vp/Vs RTM solution (indicated by yellow arrows in Figure 4c). On the other hand, the $\mathrm{Vp} / \mathrm{Vs}$ dynamic range obtained from LSRTM gathers is more consistent throughout the section, with improved layer continuity (highlighted by blue arrows in Figure 5c). These observations demonstrate the presence of non-geological, illumination variations in the pre-salt elastic properties estimated from the RTM gathers and further reiterate the importance of compensating for these variations to perform accurate reservoir characterization. 

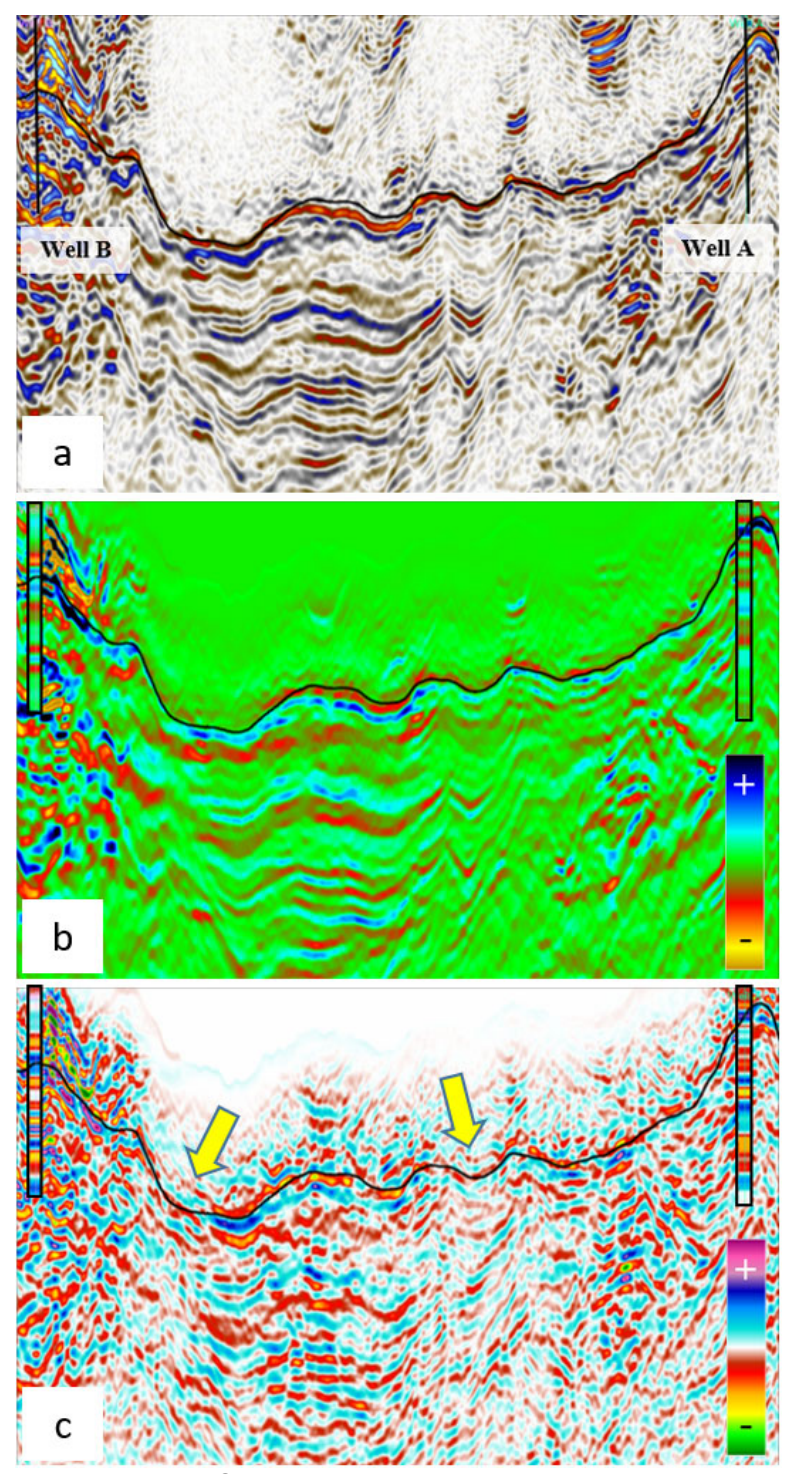

Figure 4 - AVO inversion on RTM seismic data: seismic stack (a); relative acoustic impedance (b), and relative $\mathrm{Vp} / \mathrm{Vs}$ ratio (c).

\section{Conclusions}

The image-domain LSRTM process proved to be an efficient technique to handle the illumination variations caused by complex overburden and shortcomings of the acquisition. In this study, LSRTM was applied to prestack SOGs from NAZ seismic data acquired in the Santos Basin. The benefits of the prestack application include, but are not limited to, better focusing of the fault structures, enhanced signal-to-noise ratio in the offset gathers, and compensation for illumination-related amplitude distortions with offset. Prestack LSRTM can, potentially, have a significant impact on subsequent quantitative interpretation, enhancing accuracy in elastic properties and providing foundations for more informed decisions in well placement and oil field development.
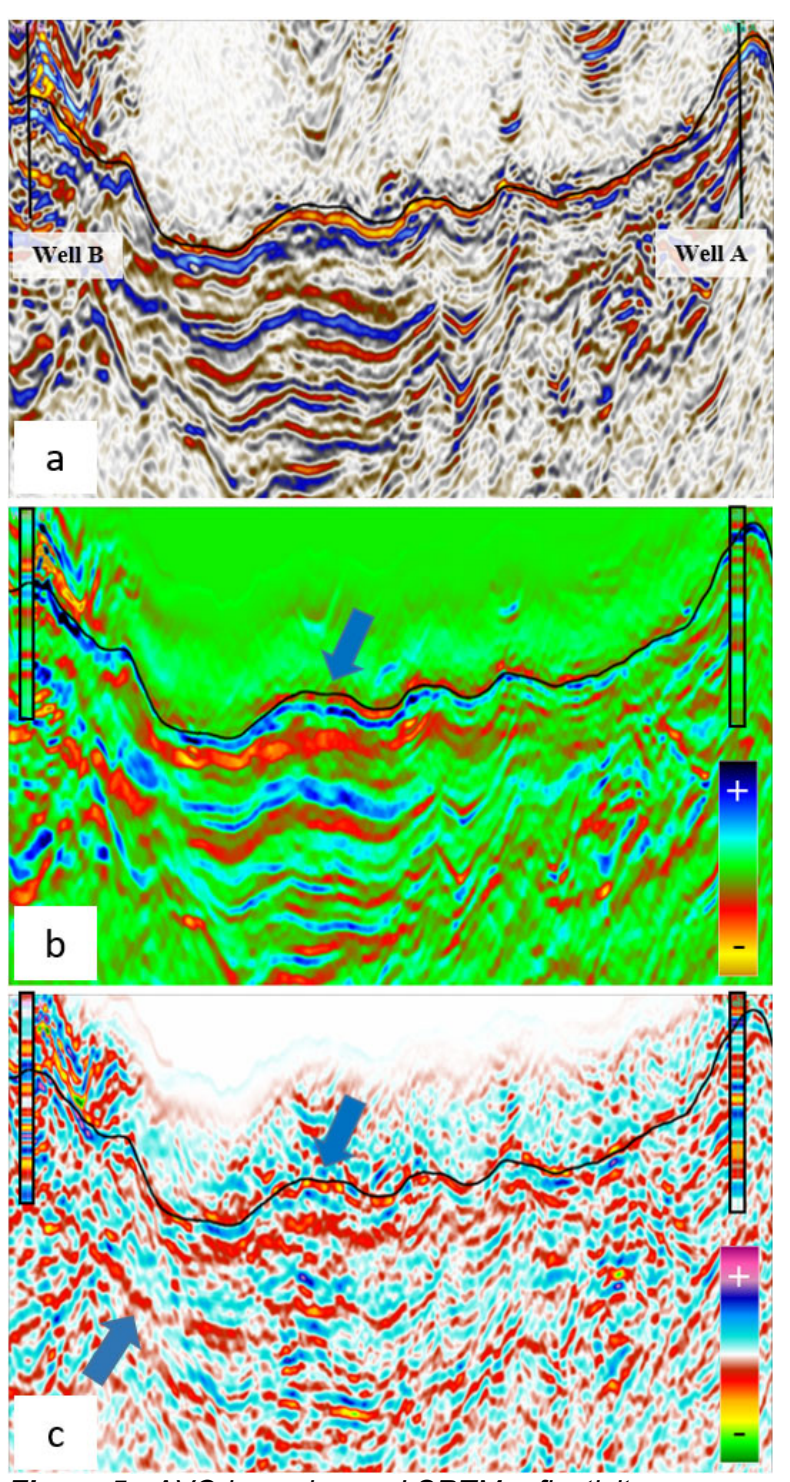

Figure 5 - AVO inversion on LSRTM reflectivity:

reflectivity stack (a); relative acoustic impedance (b), and relative $\mathrm{Vp} / \mathrm{Vs}$ ratio (c).

\section{Acknowledgments}

The authors thank Petrobras for permission to publish this study and Petrobras Processing and Research Centers together with Shell and Total staff for their contribution to the project. We thank Schlumberger for permission to submit this work and Wouter Gerrit Brouwer for his continuous support.

\section{References}

Cavalca, M., R. P Fletcher, and D. Nichols, 2018, Imagedomain least-squares migration in rapidly-varying media: Practical considerations: FirstEAGE/SBGf Workshop on Least-Squares Migration, 1-5.

Cavalca, M., R. Fletcher, and R. Bloor, 2019, Experiences of image-domain least-squares migration for quantitative interpretation: 16th InternationalCongress of the Brazilian Geophysical Society, SBGf, Expanded Abstracts, 1-6. 
Dias, R., J. Fonseca, A. Bulcão, B. Dias, L. Teixeira, A. Maul, and F. Borges, 2019, Salt stratification and least square migration to improve pre-saltreservoir images: Santos Basin, Brazilian Offshore example: 81st EAGE Conference and Exhibition, 1-3.

Fletcher, R. P., S. Archer, D. Nichols, and W. Mao, 2012, Inversion after depth imaging: 72nd Annual International Meeting, SEG, Expanded Ab-stracts, 1-5, doi: https://doi.org/10.1190/segam2012-0427.1.

Kang, W., M. Ortin, J. Monteiro, M. Fagundes, O.

Zdraveva, D. Vigh, and M. Shadrina, 2019, Improving presalt image with reflection full-waveform inversion in

Santos Basin: First EAGE Workshop on Pre-Salt

Reservoir: From Exploration to Production, 1-5.

Penna, R., S. Araújo, A. Geisslinger, R. Sansonowski, L. Oliveira, J. Rosseto, and M. Matos, 2019, Carbonate and igneous rock characterizationthrough reprocessing, FWI imaging, and elastic inversion of a legacy seismic data set in Brazilian presalt province: The Leading Edge, 38,1119,doi: https://doi.org/10.1190/tle38010011.1.

Pereira-Dias, B., C. Guerra, A. Bulcão, and R. de M. Dias, 2019, Exploring inversion strategies in image domain least squares migration: First EAGE/SBGf Workshop on Least-Squares Migration, 1-5. 